\title{
Examen de quelques difficultés rencontrées dams l'utilisation des boucles d'essais de turbomachines hydrauliques
}

\author{
Discussion of difficullites encountered \\ in the use of hydraullic turbomachine test loops
}

\author{
Robert Bonnafoux et René Bourdin \\ Département Machines et Structures \\ Direction des Études et Recherches \\ Électricité de France (Chatou)
}

\section{Introduction}

Il y a déjà bien des années que l'on effectue des essais sur boucle de turbomachines hydrauliques, à l'échelle industrielle ou sur modèle, et que l'on s'efforce d'interpréter les résultats obtenus, et l'on pourrait croire que cet art n'a plus de secrets pour ses adeptes. Il semble au contraire que, malgré les progrès importants faits dans la compréhension des phénomènes hydrauliques et hydromécaniques et dans les méthodes d'investigation, ou plutôt à cause même de ces progrès et de l'intérêt croissant qu'ils ont suscité pour de nouveaux aspects du comportement des machines, il semble donc qu'à côté de difficultés maintenant bien connues apparaissent ou se précisent de nouveaux problèmes liés à la conception de la boucle d'essais, aux interactions possibles entre la machine essayée et certains composants de l'installation ou à la caractérisation et à l'interprétation des phénomènes.

Bien qu'il puisse paraitre une gageure de vouloir faire en quelques mots le tour de tous les problèmes rencontrés, nous allons essayer de faire le point sur les principales difficultés auxquelles se heurtent tous les exploitants de boucles d'essai.

\section{Finalité des essais}

Les installations d'essais étaient généralement, jusqu'à ces dernières années, sensiblement différentes selon qu'elles étaient destinées à la recherche ou à la réception de machines. Cependant, ces différences ont maintenant tendance à s'estomper et cela, semble-t-il, pour deux raisons principales :

- L'utilisateur futur de la machine s'intéresse à des aspects toujours plus détaillés de son comportement, notamment dans les régimes transitoires. Cette attitude est aussi liée au fait que les machines sont appelées de plus en plus fréquemment à fonctionner en dehors du régime nominal, ce qui donne beaucoup plus d'acuité aux problèmes de fonctionnement à des régimes autrefois peu étudiés.

- Dans le même temps, les progrès ont été tels dans le domaine de l'acquisition des mesures et de leur traitement qu'il est devenu possible et même courant de mettre en cuvre pour des essais de réception des appareillages réservés autrefois à la recherche.

C'est ainsi que dans un programme d'essais de réception sur modèle de turbine hydraulique, il n'est pas rare que le client demande des mesures telles que les fluctuations de pression, de couple ou de poussée, la mesure et l'analyse spectrale des contraintes et des vibrations, etc...

C'est donc dans une optique beaucoup plus large que se pose maintenant le problème de la représentativité des essais sur boucle. Si les conditions propres à la machine, surtout lorsqu'il s'agit d'un modèle réduit, ne sont pas ici notre propos, quelle attitude faut-il adopter quant à la conception de la boucle d'essais et aux conditions d'installation de la machine? Faut-il reproduire les conditions réelles d'exploitation, préoccupation première du futur exploitant de la machine, ou faut-il se placer dans des conditions idéales telles que les circuits normalisés que définissent les codes d'essai de pompes [4] et ainsi mieux caractériser la machine en elle-même ? Il est évident que la réponse à ces questions ne pourra être la même lorsqu'il s'agit d'une turbine construite sur mesure pour un site déterminé que lorsqu'il s'agit d'une pompe de catalogue dont l'environnement n'est pas connu à l'avance. 


\section{Détermination des courbes caractéristiques}

\section{Etude des faibles débits}

La réduction du débit s'accompagne, en deçà d'une certaine valeur, de l'apparition de recirculations et de décollements au niveau de la roue. L'énergie libérée provoque alors des instabilités et des vibrations de la machine qui rendent les mesures difficiles. Les enregistrements présentés sur la figure 1 , ont été réalisés par le CETIM. Ils montrent bien l'accroissement des fluctuations de pression différentielle d'une pompe lorsqu'on diminue le débit. Ces phenomènes ont été largement décrits dans le document [1] établi par le groupe de travail de la SHF sur les fonctionnements des turbomachines à débits partiels. Le cas s'est présenté plusieurs fois lors d'essais faits par EDF, notamment pour une pompe hélico-centrifuge dont la caractéristique présentait un creux entre 40 et $55 \%$ environ du débit nominal. Les vibrations dans cette zone étaient telles qu'elles interdisaient pratiquement toute stabilisation du point d'essai, ce qui bien sûr a rendu difficile le tracé exact de la caractéristique dans cette zone.

\section{Machines présentant une hystérésis à certains régimes}

Les difficultés décrites ci-dessus augmentent lorsque la vitesse spécifique de la machine essayée s'accroît. Les courbes caractéristiques de pompes hélico-centrifuges et hélices ou de pompes-turbines présentent souvent à des débits inférieurs au débit nominal une zone d'hystérésis, que l'on cherche à connaître de plus en plus finement pour s'assurer du bon fonctionnement de la machine à débit partiel et pour étudier son comportement lors des transitoires (démarrages, arrêts, ...). Les essais dans cette zone, toujours délicats à cause des vibrations et fluctuations citées précédemment, peuvent être influencés par la configuration du circuit d'essais. Les paramètres qui interviennent sont :

- La forme de la courbe caractéristique du circuit : les schémas de la figure 2 tirés de [2] illustrent bien ce phénomène.
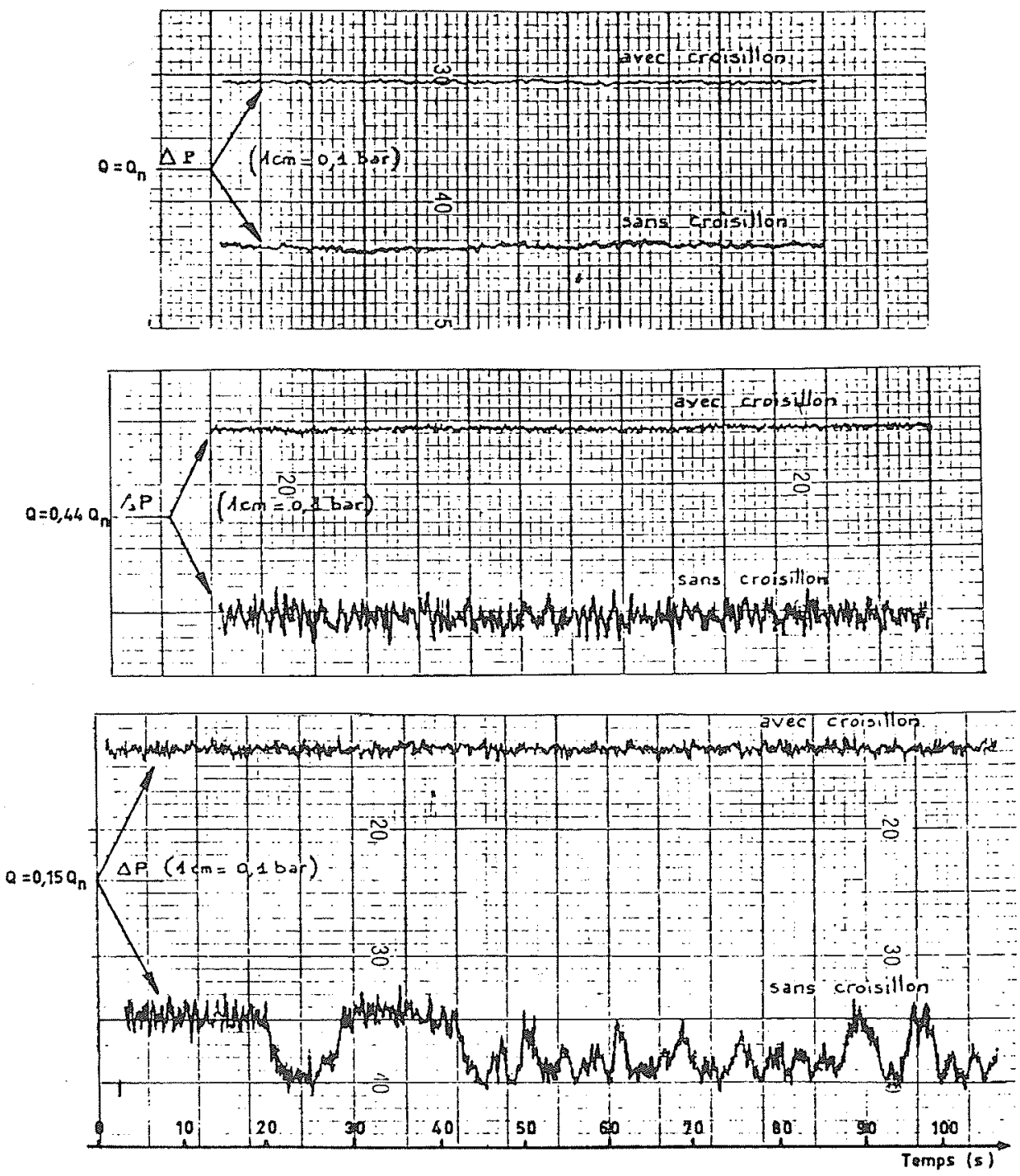

Figure 1. - Fluctuations de pression différentielle en fonction du dèbit. - Influence d'un croisillon à l'aspiration. Vitesse de la pompe : $1450 \mathrm{tr} / \mathrm{mn}$. 


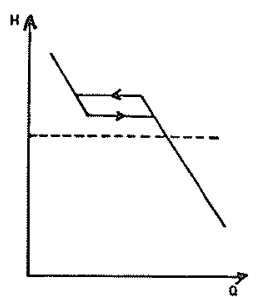

a) Horizonatale

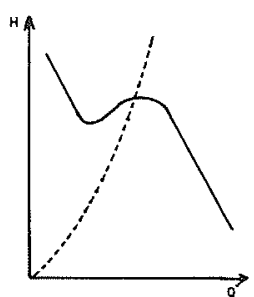

b/ Type "perte de charge"

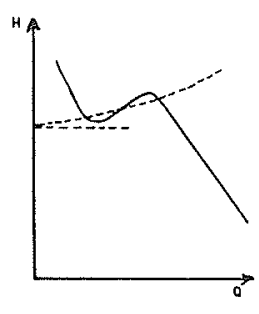

ef Type "haut cur statique
+pertec de charge"
Figure 2. - Influence de la courbe caractéristique du circuit

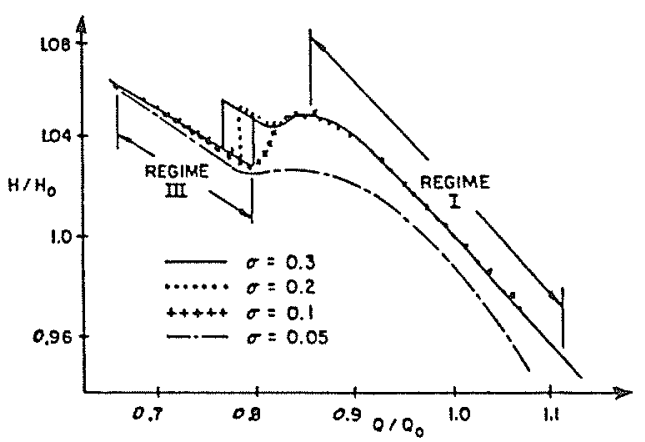

Figure 3 - Influence du sigma de l'installation (selon référence [2]).

- Le sigma de l'installation : la figure 3 tirée de [2] montre les différences obtenues dans la zone d'hystérésis lorsque la pression à l'aspiration diminue pour une pompe de rapidité spécifique :

$$
\omega_{s}=\omega \frac{Q^{1 / 2}}{(\mathrm{gH})^{3 / 4}}=0,63 \quad\left(n_{s}=35\right)
$$

On conçoit que pour l'essai de ce type de machine, il est nécessaire d'avoir une installation qui permette de reproduire des conditions de fonctionnement voisines de celles que rencontrera la pompe sur le circuit réel tant en ce qui concerne la courbe caractéristique résistante du circuit que les conditions de pression à l'aspiration de la pompe.

\section{Influence des conditions d'aspiration}

Il est bien connu que non seulement la pression, mais aussi la forme de l'écoulement influencent les performances de la machine. C'est le cas notamment des pompes hélices sensibles à la position de la tulipe d'aspiration dans le bassin. Une étude a ainsi été faite à Chatou sur une pompe hélice à volute de rapidité spécifique

$$
\omega_{s}=3,5 \quad\left(n_{s}=188\right),
$$

et a porté sur l'influence des paramètres suivants :

a) allongement de la chambre d'aspiration,

b) présence d'une marche derrière la tulipe,

c) rétrécissement symétrique de la chambre,

d) dissymétrie de l'alimentation favorisant ou contrariant la prérotation,

e) présence d'un cône sous la tulipe,

f) modification du bourrelet de la tulipe.
La modification la plus importante a été observée lors de l'essai $d$, avec une augmentation de la hauteur fournie lorsque la prérotation est contrariée, une diminution lorsque la prérotation est favorisée (fig. 4).

D'une façon plus générale, la prérotation à l'aspiration de la pompe en essai est fortement influencée par la géométrie de cette aspiration. De nombreux travaux (dont certains sont cités dans la bibliographie [1]) ont été consacrés à l'influence de la présence à l'aspiration de la pompe d'éléments tels que les croisillons anti-vortex, les guide-eau, les coudes, ... La figure 1 déjà citée montre combien un croisillon placé à l'aspiration de la pompe permet de réduire l'amplitude des fluctuations de pression différentielle lorsqu'on diminue le débit, mais il ne faut pas perdre de vue qu'un tel dispositif peut beaucoup modifier la caractéristique de la pompe, et surtout la hauteur à débit nul.

\section{Essais de cavitation}

L'étude de la cavitation a toujours posé et pose encore de nombreux problèmes, que nous avons classés ici en trois catégories :

- influence de la configuration du circuit d'essai,

- influence des conditions d'essais,

- problème de la similitude de la cavitation.

\section{Configuration du circuit d'essais}

Les résultats d'essais de cavitation peuvent être sensiblement différents, et ce pour un certain nombre de raisons liées à la configuration de circuit :

\section{Circuit ouvert ou fermé}

Le cas idéal du circuit ouvert est celui où l'aspiration et le refoulement de la pompe se font dans deux réservoirs séparés, ce qui est rarement réalisé. On se rapproche beaucoup de ce cas lorsque le réservoir d'aspiration et de refoulement est unique mais suffisamment grand pour empêcher tout rebouclage des ondes de fluctuation et permettre d'avoir une eau exempte de bulles. Lorsque divers impératifs (encombrement, aspect financier, ...) empêchent de construire une citerne suffisamment grande, on peut adopter la solution du circuit fermé, à condition d'insérer en amont de l'aspiration de la pompe un résorbeur d'air et un réservoir à surface libre, permettant de renvoyer à la pompe une eau non chargée en bulles et de découpler les perturbations engendrées dans le circuit par la machine en essai ou par tout autre organe.

\section{Présence d'organes perturbateurs de l'écoulement}

Cette présence est surtout sensible dans le cas du circuit fermé où les perturbations engendrées par ces organes (vibrations, cavitation des diaphragmes, vannes,...) sont sans cesse recyclées dans la pompe en essai.

Un exemple très significatif de ces problèmes est celui d'une pompe d'aspersion d'enceinte nucléaire essayée à Gennevilliers. Cette machine était munie d'une roue à deux ouies, et avait une rapidité spécifique $\omega_{s}=0,29$ 


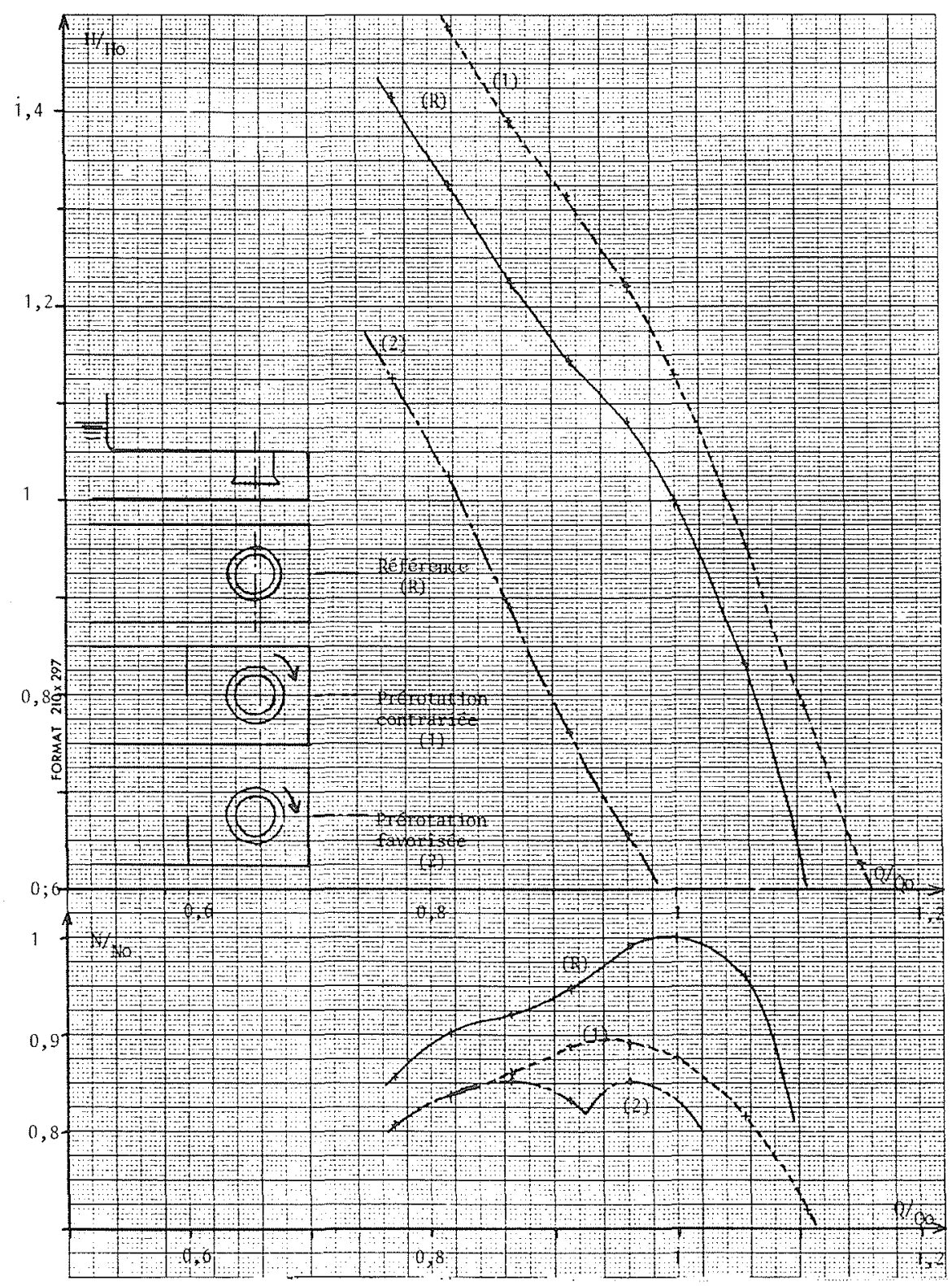

Figure 4 - Influence des conditions d'aspiration sur les caracté-

ristiques d'une pompe hélice $\left(\omega_{s}=3,5 ; n_{s}=188\right)$.

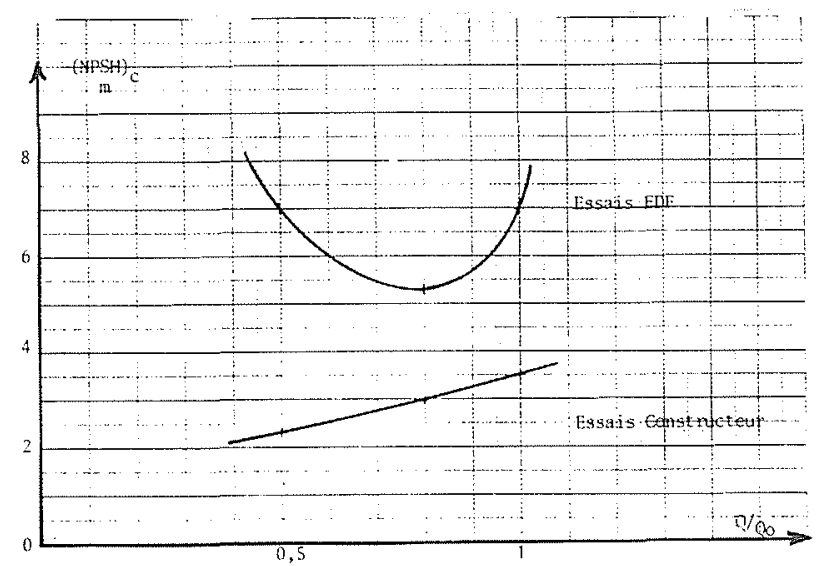

Figure 5 - Courbes de NPSH de la pompe d'aspersion essayée à Gennevilliers $\left(n_{s}=15,3\right)$ par oeillard. Les essais de cavitation réalisés chez le constructeur en boucle ouverte et à Gennevilliers en circuit fermé ont donné des résultats très différents (fig. 5).

Le constructeur disposait d'une vaste citerne (fig. 6a), assurant une eau probablement saturée en air dissous, mais exempte de bulles; la pression à l'aspiration était réglée par une vanne papillon à l'amont de la pompe, qui, semble-t-il, ne créait pas trop de perturbations de l'écoulement.

L'installation EDF était par contre un circuit fermé muni d'un diaphragme détruisant une bonne partie de l'énergie de la pompe et d'une vanne double siège assurant le réglage du débit (fig. $6 \mathrm{~b}$ ).

Ce circuit, relativement court, n'offrait donc pas de surface libre permettant le dégagement des bulles d'air (la bâche était utilisée uniquement en tampon et lors de 


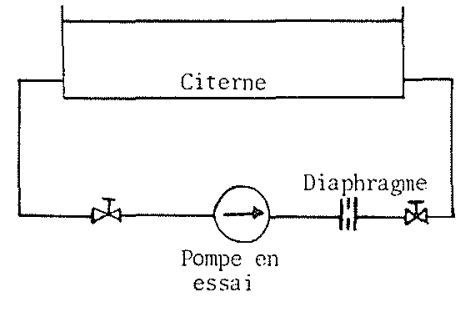

a) Constructeur

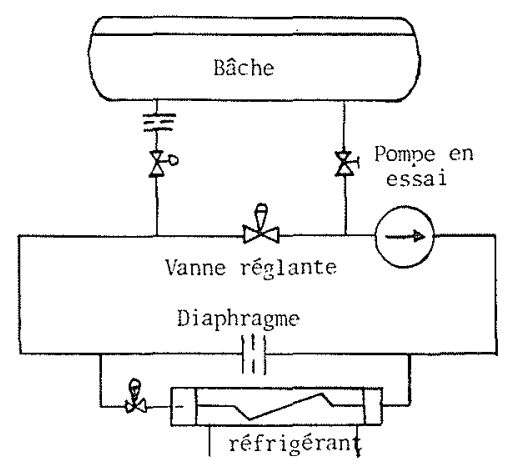

b) EDF

Figure 6. - Installations d'essais de la pompe d'aspersion

certains essais de choc thermique) et l'élimination des germes de cavitation engendrés par la pompe elle-même et par le diaphragme ou la vanne réglante. Cela expliquerait, du moins en partie, les différences trouvées.

Le même type de problème a été rencontré lors des essais d'une maquette de pompe primaire PWR $900 \mathrm{MW}$. Les essais de Chatou effectués en boucle fermée munie d'un réservoir à surface libre ont donné des valeurs de NPSH inférieures de $70 \%$ à celles trouvées par le constructeur sur boucle fermée possédant un diaphragme et des vannes entre le réservoir et l'aspiration de la pompe.

Il paraît donc important que la conception du circuit d'essais se rapproche le plus possible du circuit industriel, ce qui à la limite conduirait à prévoir une boucle particulière pour chaque type de machine.

\section{Difficultés de visualisation}

L'interprétation des figures de cavitation observées en particulier lors d'essais sur modèle est très délicate et requiert une grande expérience. Elle dépend beaucoup de la position du hublot d'observation par rapport à l'entrée de la roue. Les figures elles-mêmes sont très influencées par des différences même minimes de la géométrie tant à l'aspiration qu'au refoulement ou dans la traversée de la machine. On cite ainsi le cas où une amorce de figures de cavitation était provoquée par la seule présence de chiffres repères peints sur les aubes.

\section{Influence des conditions d'essais}

\section{Nature du fluide}

L'aspect thermodynamique de la cavitation dépend bien sûr de la nature du fluide employé [3], et rend déli- cate la transposition d'essais réalisés en eau (pour raisons de simplicité ou d'économie) au fonctionnement de la machine avec un autre fluide (cas des pompes à sodium Super-Phénix essayées en eau à Gennevilliers), mais nous ne pouvons dans le cadre de cet article nous étendre sur un sujet aussi vaste.

\section{Température du liquide}

Elle intervient non seulement sur l'aspect thermodynamique de la cavitation (variation des caractéristiques du fluide en fonction de la température [3]) et par là sur le comportement de la pompe (augmentation du NPSH avec la températue, voir par exemple [8]), mais aussi sur la précision des résultats obtenus. La tension de vapeur, intervenant dans le calcul du NPSH, augmente beaucoup avec la température, et surtout ses variations en fonction de la température augmentent quand celle-ci s'accroît, ce qui nécessite alors une excellente régulation de température, parfois difficile à maîtriser, ou à tout le moins une mesure très précise de celle-ci.

\section{Méthodologie d'essais}

Elle est souvent liée à la configuration du circuit. Le réglage de la pression par vannage à l'aspiration est toujours moins bon pour l'écoulement que la mise en dépression de l'atmosphère au-dessus de la surface libre du réservoir d'aspiration, quand celui-ci existe bien entendu.

De même, le réglage de la pression d'aspiration de la pompe en essai avec une pompe de gavage peut avoir une très grande influence comme le montre la figure 7 . Une même pompe, (rapidité spécifique $=1,69 ; n_{s}=89,2$ ) a donné des résultats de NPSH sur boucle fermée inférieurs de plus de $50 \%$ à ceux obtenus sur un autre banc d'essais avec une pompe de gavage.

D'autre part, la procédure d'essai [4] dépend de la nature de la pompe à essayer : les essais de cavitation à NPSH constant, avec balayage de la plage de débit, sont utilisables de préférence pour les machines centrifuges présentant une chute nette de caractéristiques. Pour les pompes de plus grande vitesse spécifique, où le point de décrochement est moins net, il est préférable de procéder à débit constant par diminution de la pression d'aspiration. Mais ces remarques sont plus ou moins vraies selon le critère choisi pour caractériser la cavitation.

\section{Problèmes de similitude}

Nous ne parlerons pas ici des problèmes de similitude géométrique traités dans une communication précédente. Nous n'aborderons que les problèmes relatifs à la teneur en germes de l'eau utilisée et à la transposition du NPSH en fonction de la vitesse de rotation.

\section{Teneur en germes de cavitation (cavitation à bulles)}

Ce paramètre a une grande influence sur les valeurs de NPSH trouvées aux essais, et des machines se sont revélées fonctionner en cavitation lors de la réception sur site alors que les essais sur modèle semblaient garantir un fonctionnement sans cavitation sous le NPSH du site. Des études, faites notamment par F. Danel et $Y$. Lecoffre [5], ont montré que dans la zone de cavitation, la 


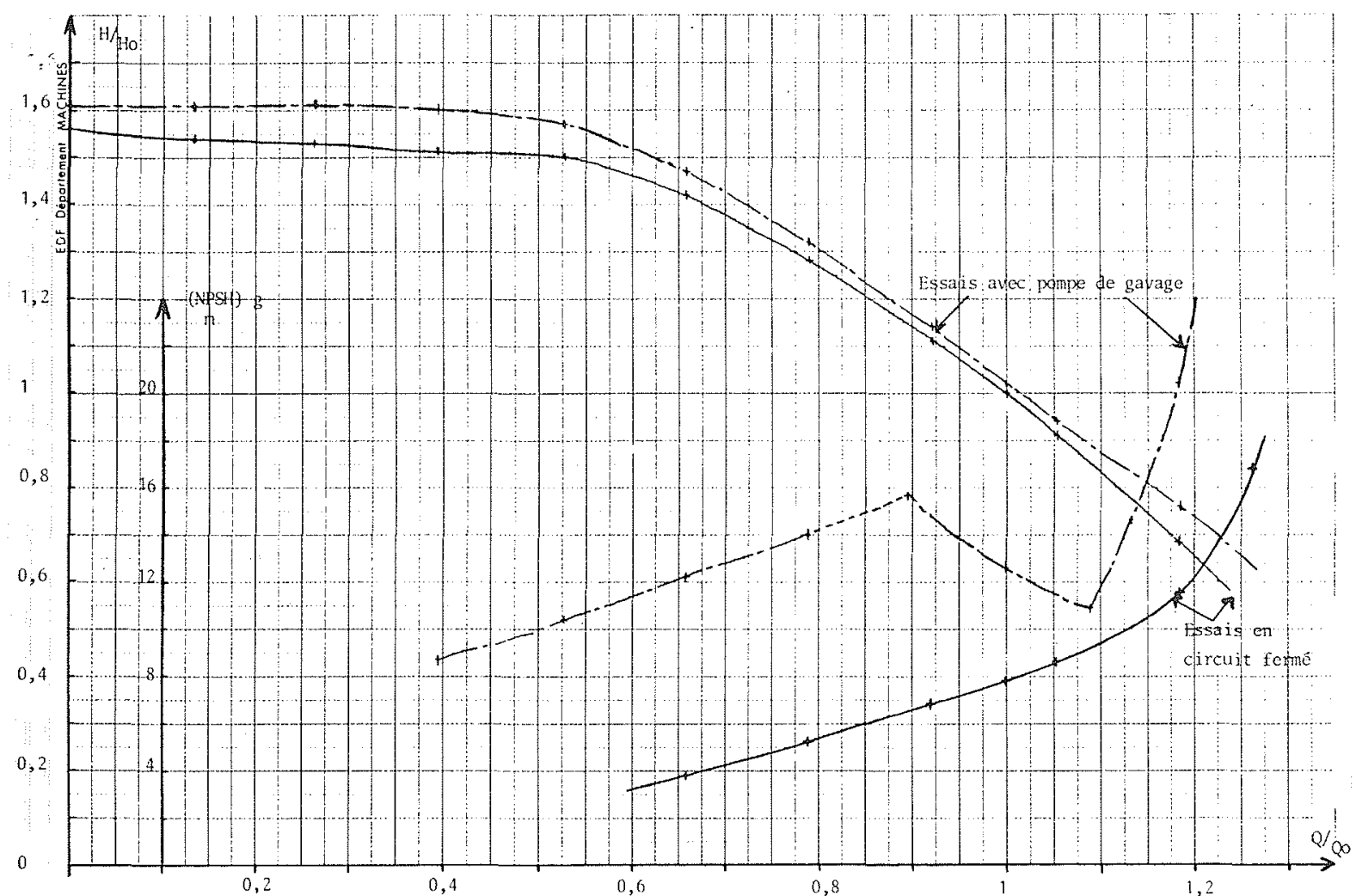

Figure 7. - Influence de l'installation sur les essais de NPSH.

Essais en circuit fermé.

- - Essai avec pompe de gavage.

pression est fonction non seulement de $\sigma$ mais aussi de la vitesse et du nombre de bulles à un instant donné, ces bulles ayant une pression critique donnée (pression à laquelle le germe doit être soumis pour être déstabilisé et connaitre une évolution explosive). La conclusion de cette étude est que pour obtenir la similitude géométrique de la cavitation sur deux profils semblables, il faut d'une part, imposer le paramètre de Thoma, d'autre part avoir à un instant donné le même nombre de bulles en expansion sur le profil, ce qui, pour un modèle à l'échelle $1 / 10$ conduit à une teneur en bulles de l'eau d'essai égale à 1000 fois celle de l'eau réelle. Le problème est ensuite de contrôler cette teneur en bulles. Un système de production et d'injection de bulles dans l'écoulement a été mis au point par Lecoffre [6], le contrôle du nombre de bulles pouvant être fait de plusieurs façons, en particulier avec un venturi de mesure de germes, décrit en [7]. L'I.M.H. de Lausanne envisage d'expérimenter prochainement ces dispositifs.

\section{Transposition du NPSH en fonction de la vitesse de rotation}

Il est généralement admis que le NPSH d'une pompe (défini par une chute de $3 \%$ de la hauteur) varie comme le carré de la vitesse de rotation, tout au moins dans une gamme que les codes d'essais [4] fixent à $\pm 20 \%$ de la vitesse nominale et pour des débits de 0,5 à 1,2 fois le débit nominal.

Il faut cependant signaler qu'une étude est actuellement en cours au CETIM qui pourrait faire penser que l'exposant peut être sensiblement différent de deux et varier non seulement d'une pompe à l'autre mais aussi, pour la même pompe, en fonction du débit. Ceci mettrait évidemment en cause la validité d'essais effectués à vitesse réduite (cas des moteurs $60 \mathrm{~Hz}$ essayés sous $50 \mathrm{~Hz}$ ). Il ne faut cependant pas oublier que toutes les causes d'imprécision dans la détermination du NPSH rendent très délicates de telles comparaisons.

\section{Comportement dynamique de la machine}

Cette étude est souvent délicate à cause des interactions entre la machine et le circuit d'essais, comme le montrent d'autres communications présentées au cours de ces Journées.

\section{Conditions $d$ 'installations}

La machine est rarement installée de façon identique sur le banc d'essai et sur le circuit réel. Supports, accouplements... sont très souvent différents. On peut citer ici le cas de plusieurs pompes que nous avons eu l'occasion d'essayer récemment :

- Pompe d'aspersion : support de béton en centrale, charpente métallique boulonnée à Gennevilliers.

- Pompe primaire de réacteur PWR : la pompe est montée sur béquilles en centrale et peut se déplacer en fonction des dilatations des tuyauteries, alors qu'elle constitue le point fixe de l'installation d'essais de Gennevilliers. 


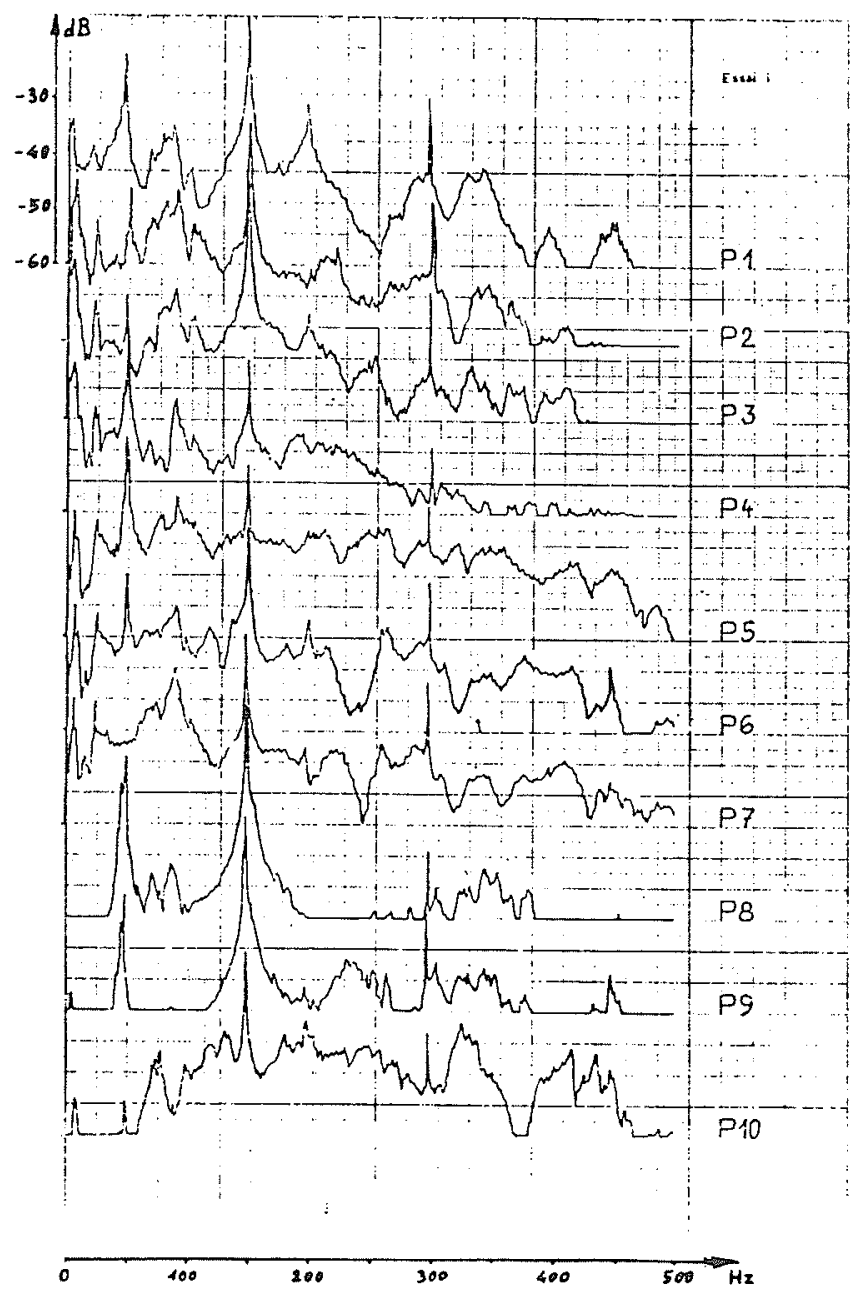

Figure 8. - Fluctuations de pression en différents points de la boucle d'essais de pompe d'aspersion.

- Pompe primaire du réacteur Super Phénix : le support de la pompe en centrale est la dalle en béton du réacteur ; pour les essais en eau de Gennevilliers, ce support est un double cône métallique, et les études préliminaires de vibrations, ont conduit à concevoir un absorbeur de vibrations à fixer sur le moteur en cas de problèmes trop importants à certaines vitesses. Il est évident que dans ces conditions la transposition au comportement in situ des observations faites sur boucle d'essais doit être entourée de beaucoup de précautions.

\section{Rebouclage des ondes en circuit fermé}

Lorsque pour des raisons d'encombrement, d'économie, ... le circuit d'essais est un circuit fermé, il peut s'établir une résonance entre la machine en essai et le circuit. D'une part celui-ci peut présenter une longueur critique vis-à-vis de la vitesse de propagation des ondes de pression, d'autre part il peut y avoir interaction entre la machine en essai et un élément du circuit : organe de perte de charge (diaphragme, vanne, déchargeur, ...), booster, etc... Le fonctionnement de la machine peut s'accompagner de fluctuations et vibrations importantes et le problème est alors de prévoir ce que sera ce fonc- tionnement sur le circuit réel. Pour cela il serait nécessaire de dégager lors des essais ce qui est dû à la pompe et ce qui est dû au circuit, et comme le montre ci-après l'exemple de la pompe d'aspersion déjà citée, ce problème est très complexe et encore mal maitrisé.

Comme nous l'avons vu précédemment, il n'y a pratiquement aucune ressemblance entre le circuit d'aspersion en centrale et le circuit d'essais de Gennevilliers : les supportages sont différents, de même que les tracés des tuyauteries. En centrale, la pompe est sur un circuit ouvert avec des pertes de charge bien réparties, alors que sur le circuit fermé de Gennevilliers, ces pertes de charge sont concentrées en deux points : diaphragme et vanne (cf. figure 6). La pompe s'est révélée fonctionner à Gennevilliers avec un niveau global de vibration (relevé à proximité des roulements) anormalement élevé. Que prévoir dans ces conditions pour le fonctionnement de la pompe en centrale?

Une étude a été réalisée pour tenter de séparer ce qui venait de la pompe et ce qui était dû au circuit. Ce dernier a été équipé de capteurs de fluctuations de pression et d'accéléromètres de part et d'autre de la pompe et des différentes singularités pouvant entraîner des perturbations de l'écoulement (diaphragmes, vanne, bâche,...). Une campagne d'essais a permis de faire un certain nombre d'enregistrements de ces signaux (fig. 8). Le dépouillement a été réalisé suivant trois directions principales :

- Analyse harmonique des fluctuations de pression, comportant l'étude analytique des spectres, l'examen de la répartition de l'énergie vibratoire dans la boucle, l'évolution des fluctuations en fonction du débit, l'étude des intercorrélations et du déphasage entre les signaux des divers capteurs, la recherche d'une éventuelle résonance acoustique du circuit.

- Etude des transitoires hydrauliques.

- Recherche d'un couplage hydroélastique par analyse spectrale des accélérations et étude des intercorrélations entre capteurs de pression et de vibration. Cette étude a permis de tirer les conclusions suivantes:

- Les raies de vibrations les plus énergétiques correspondent à des fréquences de $149 \mathrm{~Hz}$ (fréquence de passage des aubes) et surtout $50 \mathrm{~Hz}$, alors que le $25 \mathrm{~Hz}$ (vitesse de rotation de la pompe) est négligeable.

- Le diaphragme crée essentiellement une turbulence concentrée dans les basses fréquences.

- La vanne engendre par contre une turbulence qui s'étale sur la gamme [100-300 Hz].

- L'amplitude de la raie à $50 \mathrm{~Hz}$ semble résulter d'une résonance du circuit, car elle n'est pas fonction de la vitesse de rotation (comme l'a montré l'enregistrement de démarrages-arrêts).

Comme on le voit, cette longue étude n'a pas permis de déterminer avec précision l'origine de la fréquence de vibration de $50 \mathrm{~Hz}$. On ne peut, avec les enregistrements faits, que suspecter une résonance du circuit ; dans ce cas on peut espérer un bon comportement de la pompe sur le circuit d'aspersion (ouvert) de la centrale.

Des études sur les divers mécanismes de génération, de transfert et de rayonnement de l'énergie vibratoire sont encore nécessaires pour rendre opérationnel, à 
partir d'un nombre optimal de capteurs, un éventuel système de contrôle et de prévision des vibrations. De telles études sont actuellement en cours dans plusieurs organismes.

\section{Conclusion}

Pour terminer ce survol nécessairement bien rapide des difficultés auxquelles se heurtent les exploitants de boucles d'essais de turbomachines hydrauliques, il parait nécessaire de souligner que, si les progrès récents dans l'acquisition et le traitement des mesures permettent d'accéder à de plus en plus de phénomènes, il n'en reste pas moins qu'il serait vain de vouloir faire dire à une installation et à une machine d'essais plus qu'elles ne peuvent le faire. La conception d'un modèle réduit de pompe ou de turbine, comme celle d'une boucle d'essais, exige souvent certains choix qui l'empêchent d'être représentatif dans tous les domaines, ces choix étant aussi bien techniques qu'économiques.

Tirer tout le parti possible de son installation, mais aussi ne pas se laisser entraîner au delà de certaines limites, sont donc les deux aspects du rôle de l'exploitant.

Nous voudrions conclure en exprimant notre gratitude aux diverses personnes qui nous ont fait profiter de leur expérience en ce domaine. Qu'elles appartiennent à la Société Bergeron, au CETIM Nantes, à la Société
Neyrpic ou à l'Institut de Machines Hydrauliques de Lausanne, le concours qu'elles nous ont aimablement apporté nous a été précieux.

\section{Références}

[1] SOCIETE HYDROTECHNIQUE DE FRANCE. - Division "Applications Industrielles de la Mécanique des Fluides" Groupe de travail $\mathrm{n}^{\circ} 1$ : "Etude des fonctionnements à débit partiel des turbomachines à faible variation volumique du fluide". Document de travail : cahier $\mathrm{n}^{\circ} 1$ : texte ; cahier $n^{\circ} 2$ : figures; cahier $n^{\circ} 3$ : bibliographie.

[2] FISHER R.K. and WEBB D.R. - Effect of cavitation on the discontinuity point and on alternating pressures and gate torques on a pump-turbine model in the pump cycle. Sympo. sium AIRH, Fort-Collins, U.S.A., 12-14 juin 1978.

[3] PEARSALL I.S. - Cavitation. Lecture series "Off design performance of pumps". Von Karman Institute for Fluid Dynamics, 6-8 th march 1978.

[4] NF X 10-602 (ou ISO 3555 ) - Pompes centrifuges, hélicocentrifuges et hélices. Code d'essais de réception classe $B$ (1977).

[5]DANEL F. et LECOFFRE Y. - Influence des germes contenus dans l'eau sur la cavitation développée à bulles séparées. Symposium AIRH, Grenoble 1976.

[6] LECOFFRE Y, - Contrôle de la nucléation d'une eau en moyen d'essais. Symposium AIRH, Leningrad 1976.

[7] LECOFFRE Y. et MARCOZ J. - Essais en cavitation à bulles séparées. Méthodes de contrôle. Venturi de mesure de germes. La Houille Blanche, $\mathrm{n}^{\circ}$. 5, 1978.

[8] FLORJANCIC D. - Influence de la température de l'eau sur la capacité d'aspiration des pompes. Numéro de Recherches Sulzer, 1971. 


\section{Discussion}

M. le Président. - Je remercie M. BONNAFOUX.

De nombreuses critiques ont été soulevées par les auteurs. Y-a-t-il, dans la salle, des avis sur les précautions à prendre et ce qu'il faut éviter ou ne pas faire?

La revue qu'a fait $M$. BONNAFOUX portait sur la détermination des caractéristiques, la détermination des performances en cavitation, et sur le comportement mécanique et dynamique de la machine.

M. CANA VELIS. - La communication de M. BONNAFOUX semble un peu pessimiste en ce qui concerne les mesures de NPSH. Il faut quand même signaler que dans le cas de NPSH correspondant à $3 \%$ ou $10 \%$ de chute de hauteur, on obtient souvent des concordances remarquables entre essais sur modèle et machine industrielle dans les installations différentes. Les résultats sont évidemment moins satisfaisants au niveau du début de cavitation physique et d'apparition des premières bulles.

M. le Président. - Je rejoins un peu M. CANAvelis sur l'aspect pessimiste que vous avez exposé. Il y a des machines qu'on ne pourra jamais bien mesurer, et cela ne vient pas de l'installation, mais de la machine elle-même.

Je me souviens - et vous aussi certainement - d'essais que nous avons tenté de faire il y a vingt ans sur les turbines du Rhin : sur certaines de ces turbines, il n'était pas possible de faire de mesure dans une fourchette d'une dizaine de $M W$, et ce n'était pas à cause de l'installation, mais de la machine elle-même.

Il y a des machines que l'on a montées en plate-forme d'essais, et sur lesquelles on n'a jamais pu non plus faire de mesures dans certains zones de leur caractéristique du fait de l'hystérésis dont vous nous avez parlé, et qui provenait des caractéristiques des machines et non pas de l'installation.

M. GUITON. - Je voudrais faire une remarque à propos de la dernière figure présentée par M. BONNAFOUX. Elle a trait, si j'ai bien compris, à un circuit d'essai de pompe comportant un diaphragme et où une variation de vitesse de rotation de la pompe a pu montrer que les principales fluctuations de pression du circuit avaient comme source le diaphragme et non la pompe.

C'est un des moyens utilisés pour n'attribuer à la pompe que les fluctuations qui ont celle-ci pour origine.

Quand la variation de vitesse n'est pas possible, deux voies restent ouvertes:

1) Disposer un dispositif d'amortissement des fluctuations de pression entre la pompe et l'organe de décharge.

C'est par exemple un dispositif du genre de celui que $M$. ALMING a présenté au Congrès de Manchester.

Mais ces dispositifs introduisent d'autres fréquences propres plus basses dans le circuit et peuvent conduire à d'autres résonances. Il faut en tenir compte par exemple par une possibilité de modification.
2) Utiliser des méthodes d'intercorrélation dans le traitement des signaux de fluctuations de pression enregistrés simultanément à la pompe et à l'organe de décharge ou en un autre point. Ces méthodes que je connais mal permettent, je crois, de faire le juge de paix entre la pompe et l'autre organe.

Ces deux voies sont à développer

M. BONNAFOUX. - Ces recherches ont été faites dans plusieurs cas; elles sont toujours très difficiles à interpréter.

M. DOERFLER. Je voudrais proposer une raison possible au phénomène que $M$. CANAVELIS a remarqué.

Quand la cavitation est notable, elle peut peut-être découpler totalement les deux oscillations à l'aval et à l'amont. Quand on a seulement une faible cavitation, l'élasticité de la cavité est peutêtre une propriété essentielle du comportement dynamique de la boucle d'essai

M. le Président. - Il est bien certain, malgré tout, que le développement des moyens d'essais, qu'ils soient effectués sur modèle réduit ou sur machine réelle installée sur une plateforme d'essais, conduit les intéressés -client, fournisseur ou futur exploitant - à en demander toujours plus. Quand on a sous la main un outil avec lequel on peut jouer, hors de toute contrainte d'exploitation, il est tellement tentant de demander des essais qu'on ne pourra plus exécuter ultérieurement.

C'est là où, je crois, la remarque de M. BONNAFOUX prend toute sa valeur. Il faut, dans ce cas, savoir peser ce qui est réaliste et ce qui ne l'est pas, ce qui est possible et ce qui ne l'est pas.

$\mathrm{Je}$ peux vous citer des exemples de modèles, dans d'autres domaines, où, à partir du moment où celui-ci existe, on veut lui faire répondre tout ce que l'on veut savoir. Or, au départ, un modèle a été construit pour satisfaire à certaines lois de similitude et, comme tout modèle, il est forcé d'en approcher certaines et d'en oublier d'autres. Il faut être bien conscient de cela. Quand on a fait une approximation, le modèle sera capable de répondre dans les hypothèses qui ont été prises dans le cadre de cette approximation. Mais, si on veut le faire répondre hors de la gamme pour laquelle il a été conçu, il faut s'attendre à des déconvenues, que ce soit dans la manière dont la machine est montée mécaniquement par rapport à la manière dont elle sera montée sur l'installation grandeur, que ce soit dans la manière dont la dissipation d'énergie est faite, dont les bulles se résolvent ou ne se résolvent pas, tout doit être pris en compte. Il ne faut pas, aveuglément, dire : j'ai un modèle, j'appuie sur le bouton et je regarde.

Si l'on est malheureusement amené à apporter ces précisions maintenant, c'est que l'expérience montre que l'on a tendance à sortir du modèle "contrôle des performances hydrauliques ou de la cavitation" pour faire du modèle "contrôle de comportement mécanique et dynamique". Or, souvent on fait ainsi des choses qui n'ont pas de fondement théorique suffisamment solide. 